\title{
Testing for illicit drug use in mental health services
}

\author{
Abu Abraham \& Jason Luty
}

\begin{abstract}
SUMMARY
Testing for illicit drug use is used in pre-employment checks, the criminal justice system, sports medicine and for screening and confirmatory purposes in patients with physical and mental illnesses. The types of drugs tested for and the methods used vary depending on the indication. This article focuses primarily on blood, urine and oral fluids, which are specimens more commonly used in mental health settings, although hair and sweat are increasingly used in medico-legal cases and in child protection issues. The main drugs and their metabolism are discussed to gain a better understanding of the methods used and for accurate interpretation. Methods to ensure validity during sample collection are explored. False-positive and false-negative tests are common and possible confounders are discussed.
\end{abstract}

\section{DECLARATION OF INTEREST}

None.

Illicit drug use is the practice of consuming a legally restricted substance in a manner contrary to the moral or traditional practices of a particular culture. In 2005/2006, over $10 \%$ of adults between 16 and 59 years of age had used illicit drugs in the previous year (Home Office 2006). The use of 'Class A drugs' (usually heroin or cocaine) was over $3 \%$, mainly because of the rise in cocaine use. In younger adults aged between 16 and 24, illicit drug use was over $25 \%$ in the previous year; $17 \%$ of children aged $11-15$ reported taking drugs in the previous year and 9\% in the past month (Home Office 2006). The prevalence in mental health services is much higher: $25 \%$ of adult and up to $50 \%$ of adolescent in-patients in psychiatric units have an illicit drug use disorder (Crome 2006).

Testing for illicit drug use involves the analysis of biological material to detect drugs or their metabolites in the body. In mental health services it is used both to confirm diagnosis and manage care. Courts and doctors are often asked to perform and interpret the results of drug and alcohol tests for these civil proceedings.

\section{Quality control and types of drug analysis}

Drug analysis can be classified as used either primarily for screening purposes or for confirmatory purposes (Box 1). Ideally, the standard procedure should involve ahighly sensitive screening technique, followed by use of a highly specific confirmatory technique for samples identified as positive. Most screening procedures use immunoassays, which allow large-scale screening through automation and rapid detection (Armbruster 1993). They are therefore ideal for home-testing kits, nearpatient tests or point-of-care screenings, as testing the specimen, obtaining the results and interpretation can all be done near the patient without the use of specialised laboratory settings. Immunoassay techniques include cloned enzyme donor immunoassay (CEDIA), enzyme-multiplied immunoassay technique (EMIT), fluorescence polarisation immunoassay, immunoturbidimetric assay and radioimmunoassay.

When patients present with a reliable history of psychiatric symptoms and clinical signs are consistent with the history, drug testing is not requested routinely in everyday clinical practice. Even so, it is sometimes good practice to support a diagnosis with testing, especially when the patient is new to mental health services or has a history of illicit drug use. When the history is not available or if it does not match the clinical symptoms/ signs, it becomes necessary to carry out drug tests to establish a diagnosis. In the drug and alcohol

\section{BOX 1 Comparison of screening and confirmatory drug tests}

- On-the-spot screening by immunoassays: usually sufficient (even in drug and alcohol services) given a good history, and a mental state and physical examination

- Confirmatory laboratory tests: indicated if there is a diagnostic dilemma, if the patient requests such a test or disputes a screening result, or if there are serious implications to a positive result (e.g. in a drug rehabilitation requirement programme or child protection cases)
Abu Abraham is an ST5 trainee in general adult psychiatry, South Essex Partnership NHS Foundation Trust. His special interests are addictions psychiatry and mental health law. Jason Luty is consultant in addictions psychiatry with South Essex Partnership NHS Trust and an honorary consultant psychiatrist with Cambridge and Peterborough Partnership NHS Trust. He has written many research publications and editorials on substance use, including articles for Advances in Psychiatric Treatment and the BMJ. His main research interest is the stigma of mental illness in general and substance misuse in particular. Correspondence Dr Abu Abraham, ST5 in General Adult Psychiatry, South Essex Partnership NHS Foundation Trust, Mental Health Unit, Basildon Hospital, Basildon SS16 5NL, UK. Email: abu.abraham @southessex-trust.nhs.uk 
setting, testing is essential before substitute prescribing of methadone or buprenorphine. In all the above cases, a screening test is often sufficient.

However, there are situations where the patient denies the use of drugs despite positive screening tests. It then becomes important to perform a confirmatory test, as screening tests can yield false-positive results. For example, codeine is a common constituent in cold cures and crossreacts with tests for heroin. Hence the need for a confirmatory test in the form of chromatography. A confirmatory test is also required in situations where there are ominous implications for the diagnosis of illicit drug use. This is so in criminal justice, the workplace, sports medicine and child protection. Chromatographic techniques are the most accurate, specific and reliable method of testing. The disadvantage is that they are timeconsuming, expensive, require a laboratory and high level of expertise to perform. Several different types of chromatographic techniques are used in laboratories for urine drug analysis: gas chromatography-mass spectrometry (GC-MS) provides the most specific type of analytical tool. Generally, GC-MS is performed only after the screening test.

The United Kingdom Accreditation Service (UKAS) is the sole national accreditation body recognised by the government to assess, against internationally agreed standards, organisations that provide certification, testing, inspection and calibration services for drug testing. In the UK, laboratories are expected to possess a UKAS certification before providing drug-testing services.

\section{Methods}

Drug testing is a procedure involving three stages: collection of specimens, laboratory analysis and then interpretation of results based on the indication (Saxon 1988).

The types of drugs tested for and the methods used vary depending on the needs at various settings. The presence of a drug itself in a specimen does not prove illicit use. It could suggest accidental

\section{TABLE 1 Comparison of main drug-testing samples}

\begin{tabular}{|lll|}
\hline Specimen & Advantages & Disadvantages \\
\hline Urine & $\begin{array}{l}\text { Easy to administer, inexpensive and } \\
\text { instant results in near-patient tests }\end{array}$ & $\begin{array}{l}\text { Easy adulteration, need for a toilet, } \\
\text { invasive or embarrassing for donors }\end{array}$ \\
\hline Saliva & $\begin{array}{l}\text { Easy collection, donor-friendly, hard } \\
\text { to adulterate, non-invasive, no need } \\
\text { for toilet }\end{array}$ & $\begin{array}{l}\text { Detection window varies widely, } \\
\text { contamination by smoked drugs }\end{array}$ \\
\hline Blood & $\begin{array}{l}\text { Most recent use, detects 'under the } \\
\text { influence' states, hard to adulterate }\end{array}$ & $\begin{array}{l}\text { Very invasive and difficult to collect, } \\
\text { short detection window }\end{array}$ \\
\hline
\end{tabular}

or unavoidable contamination. For example, many British bank notes, which have been used by drug misusers, are contaminated with cocaine and this can be detected on the hands of bank staff. Similarly, police who raid illicit drug warehouses are likely to be contaminated by aerosols and powder. The detection of the parent drug in this case does not indicate consumption but contamination. When illicit drugs are ingested they are metabolised and the metabolites along with the parent drug are excreted in body fluids. Presence of metabolites therefore confirms consumption and not just contamination. For this reason, many tests are designed to detect the presence of drug metabolites (e.g. 6-monoacetylmorphine - a metabolite of heroin) rather than the parent drug itself.

Specimens used in mental health services for drug testing include urine, oral fluid and blood (Table 1). Other specimens such as sweat and hair are more commonly used in forensic settings and in medico-legal cases but rarely, if ever, used in mental health settings.

\section{Urine}

The most commonly used specimen in mental health settings continues to be urine. Commercial drugtesting kits can detect up to ten different drugs, which include barbiturates. Barbiturate misuse is rare and hence testing is often unnecessary unless there is a clinical suspicion of misuse. The other nine drugs include amphetamine, methamphetamine, 3, 4-methylenedioxymethamphetamine (MDMA or 'ecstasy'), cocaine, cannabis, methadone, opiates, benzodiazepines and tricyclic antidepressants.

Urine can be used for both screening and confirmatory tests. Immunoassay kits vary in cost from $£ 4$ to $£ 6$, depending on the number of kits bought and whether the panel consists of one-drug, five-drug or up to eight-drug tests. Urine drug tests are easy to administer and costs are lower than for other types of drug testing. Results are almost instant and proprietary dipstick tests (near-patient testing) mean that a laboratory or a technician is not essential. The kits are easy to store and have a long shelf life. For these reasons they are the first choice for frequent, random drug testing in mental health services.

There are, however, several drawbacks. There is often a 3-day window of detection (duration of detection after drug consumption), depending on the individual and the substance used. There is the need of a bathroom to obtain the sample. The specimen is easier to adulterate than others and therefore it may need to be taken under direct observation. This can be degrading and embarrassing (Dolan 2004). 


\section{Oral fluid}

Oral fluid is the serous transudate extracted osmotically from the buccal mucosa by the testing pad. Drug tests are donor-friendly and noninvasive. The specimen can be collected easily under direct observation in virtually any environment without the loss of privacy. It is difficult to adulterate and substitute false samples. Methods are available to give results in minutes by nearpatient tests using dipsticks or portable card readers. It is also useful when multiple serial samples are needed in child protection (Kim 2002). Child protection proceedings often require confirmation of illicit drug use or repeated confirmation of abstinence. This is particularly important in cases where the child is in care or thought to be at risk owing to parental drug misuse. It is regarded as the best method for testing very recent drug use. It is a very important tool for therapeutic drug monitoring and assessment of drug-impaired driving, especially since there is a correlation between drug concentrations in oral fluids and blood (Pehrsson 2008).

The main disadvantage of this method is the short detection window. Depending on the variation in the rate of oral fluid production or the type/density of the drug used, the detection window ranges from a few hours to $2-3$ days. Drugs that are smoked or ingested orally lead to higher concentrations because of residual amounts of the compound in the oral cavity, therefore tests may be inaccurate. It can be inconvenient to obtain samples if the patient's mouth is dry or if there is a failure to produce adequate amounts of oral fluid. Patients may also try to disrupt samples by chewing other substances.

\section{Blood}

Blood tests detect recent drug use (over the past few hours). Therefore, if an incident is suspected to be the result of drug use, the best sample to be tested near the time of the incident is the blood. This can clearly show that the person continues to be under the influence of the drug shortly after the incident. Blood tests are done mostly as part of routine investigations in patients attending mental health services, to monitor physical health.

In alcohol misuse, the individual's blood can be used to estimate blood alcohol concentrations. Breath alcohol concentrations are more convenient and a reasonable estimate of blood alcohol levels can be made from these. Blood tests are, however, not an accurate indicator of the extent of liver damage nor of the current level of drinking. For a detailed account on alcohol and blood investigation, see Drummond \& Ghodse (1999).
In drug misuse, blood tests are done to assess baseline health status at first assessment. The British National Formulary urges caution in the prescription of substitute drugs such as methadone in conditions such as hypothyroidism and renal problems. However, clinicians often fail to perform thyroid function tests and other routine blood tests before starting methadone, as complications arising from thyroid or renal disease are uncommon in otherwise healthy drug users. Blood tests also form a very important part of screening intravenous drug users for blood-borne diseases such as hepatitis and HIV.

\section{Collection of samples}

The drug concentration in specimens depends on route of administration, time since consumption and the person's physiological state. If the results are expected to come under legal challenge, the specimen is ideally handled according to "chain of custody' procedure. This is more often used in law enforcement, the workplace and sports medicine. It involves a legally defensible process that can be submitted in court as proof that the final result belongs to the patient. It involves the patient, staff member and laboratory staff signing various documents as the sample proceeds through the analysis process (Workplace Drug Testing Forum 2001). It is rarely used in mental health settings, where the procedure for the collection of samples varies with the indication and setting of the test. Specimens are always collected under circumstances that respect the individual's dignity.

\section{Accident and emergency}

In accident and emergency (A\&E) departments and Section 136 suites (places of safety for the assessment of an individual placed under Section 136 of the Mental Health Act 1983), acute presentations of psychiatric disorders are common. For a new patient or a known patient with a presentation that is not in keeping with their relapse signature, a drug screen becomes invaluable to aid diagnosis. A patient with known drug misuse can also present when they have had too much (intoxication) or too little (withdrawal) of their primary drug of misuse. Here, the accuracy of the test is less important. What is more of a challenge is the act of collection itself as many individuals can be too disturbed to issue a specimen. Clinicians should consider oral fluid collection in these instances.

\section{Acute psychiatric wards}

In acute psychiatric wards, measures are usually taken to ensure a valid sample during collection. Direct observation by staff of specimen donation is 
probably the simplest of these. Patients often resist direct observation, but a degree of supervision needs to be discussed with the patient. If this is inadvisable, oral fluid testing, in which direct observation may be perceived as less intrusive, may be used.

With the urine sample, a first morning specimen is the most concentrated and represents the ideal specimen. The donor could be requested to leave all possible belongings, including outer garments, outside the collection room. People have been known to scoop water from a commode or sink to dilute the sample. Using a urinal instead of a commode and adding a dye in the toilet water can prevent this. Placing the sink outside the urine collection area is another method to prevent dilution (Saxon 1988).

\section{Drug and alcohol services}

In drug and alcohol services, the patient group is quite diverse. There are those who are well motivated, engage well with services and have a history of giving illicit drug-free specimens for a substantial period. Here, rigorous measures to collect specimens are not indicated. Supervised collection (direct observation) is desirable for individuals living with young and vulnerable children with whom Social Services are actively involved. It is also desirable for those with poor adherence, those who have a history of tampering with specimens, those whose clinical presentation does not match presenting history, and those under probation services who are on a court order for drug testing (e.g. drug rehabilitation requirement programme). In such situations, oral fluid testing may be more appropriate as it may not always be possible to arrange for directly observed urine sample collection.

\section{Assessing integrity of samples - false negatives}

Urine tampering refers collectively to methods used to falsify a urine sample so that illicit substance use is not detected (Jaffee 2007). This yields a false-negative result. Methods of tampering fall into three categories: in vivo adulteration, which involves ingestion of a chemical before micturition (including simple dilution through the excess consumption of water); in vitro adulteration, which involves addition of a chemical to a sample after micturition; and urine substitution in which a sample from another (presumably abstinent) person is used.

\section{Validity of the specimen}

Details such as volume, appearance, colour, $\mathrm{pH}$, temperature and specific gravity can ascertain the validity of a specimen and may be tested and documented. A healthy sample is typically translucent and light yellow in appearance. It is advisable to record the temperature within $4 \mathrm{~min}$ of collection; it should be $32^{\circ} \mathrm{C}$ to $38^{\circ} \mathrm{C}$ (United States Department of Transportation 2009). The $\mathrm{pH}$ for normal urine fluctuates throughout the day, but usually is in the range of 4.5-8.0. Specimen contamination should be suspected if the $\mathrm{pH}$ level is less than 3 or greater than 11 , or if the specific gravity is less than 1.002 or greater than 1.020. Urinary creatinine concentration of less than $20 \mathrm{mg} / \mathrm{dl}$ is considered dilute, whereas concentration of less than $5 \mathrm{mg} / \mathrm{dl}$ is inconsistent with human urine. Urinary nitrite levels should be less than $500 \mu \mathrm{g} / \mathrm{ml}$ (Casavant 2002).

\section{Urine tampering}

The immunoassay kits often have a temperature strip attached to the cup. This is the most frequently used measure of the sample integrity. Other measures mentioned earlier require a laboratory to assess the urine sample and one can request a laboratory analysis if one suspects the sample to be adulterated. A simple test to determine whether substances such as soap have been added to the urine is to shake the sample. Excessive bubble formation that is long lasting can indicate an attempt to tamper with the specimen (Warner 1989). In the past, patients often added methadone mixture directly to samples to produce a positive test when they had been suspected of selling all their methadone prescription. However, most enzyme immunoassays now detect this as the preservatives in methadone mixture effectively inactivate the enzyme process and produce an invalid result that is immediately recognisable on the test strip. Also, one can test for the methadone metabolite ethylene dimethyl diphenyl pyrrolidine (EDDP) that is seen only in those who have consumed and metabolised methadone, rather than those who add it directly to their urine.

\section{Urine substitution}

Liquid drain cleaner, chlorine bleach, liquid soap, ammonia, hydrogen peroxide, lemon juice and eye drops have all been used to manipulate urine. Other products containing glutaraldehyde, sodium or potassium nitrate, peroxide and peroxidase, and pyridinium chlorochromate are sold to falsify urine specimens (Jaffee 2007). Several products, such as Intect 7, Adultacheck 4 and Mask Ultra Screen, can be used to check for the presence of adulterants (Peace 2002). They are convenient, easy-to-use dipstick devices that identify adulterants commonly associated with 
BOX 2 Drug metabolism

A basic understanding of the metabolism, major metabolites and duration of detection of various drugs helps the clinician decide:

- whether to order a screening or a confirmatory test

- whether to look for the parent drug or metabolite

- the timing of the test

- the recency of drug use

attempts to interfere with the accuracy of a drug test. Unfortunately, these kits are too expensive to be used routinely in mental health settings.

\section{Storage}

The specimen should be kept in locked storage at a temperature of $4^{\circ} \mathrm{C}$. This is to preserve its integrity. Long-term storage requires $-15^{\circ} \mathrm{C}$. In medico-legal cases, laboratories are expected to retain all samples confirmed positive for at least a year unless otherwise instructed by the customer. After this period, laboratories are required to store samples only if the customer has requested them to do so or if the samples are under legal challenge (Workplace Drug Testing Forum 2001).

\section{Drugs and their metabolism}

Understanding the metabolism of the various drugs in the body and their main metabolites is crucial in making accurate interpretations of drug test results (Box 2). The metabolism and its relevance to drug testing will now be discussed.

\section{Amphetamines}

There are a large number of synthetic, chemically related amphetamine-like compounds that are misused. Most amphetamine assays are designed to detect amphetamine and commonly misused chemical analogues such as methamphetamine, methylenedioxyamphetamine, methylenedioxyethylamphetamine and MDMA.

The amphetamine immunoassay does not distinguish between the two isomers of methamphetamine, d-methamphetamine and l-methamphetamine (l-desoxyephedrine). The former is responsible for the central stimulant effects, whereas the latter mainly works peripherally and has no euphoric effects (Eskridge 1997). L-methamphetamine is an active ingredient in some over-the-counter nasal decongestants causing false-positive results when taken in sufficient quantities. Another problem is that the sensitivity for MDMA is about $50 \%$ less than for amphetamine and methamphetamine. Tests incorporating three monoclonal antibodies specific for amphetamine, methamphetamine and
MDMA resulting in greater sensitivity for detection of MDMA should be considered if MDMA use is suspected (Hsu 2003).

\section{Benzodiazepines}

The widespread use of prescribed benzodiazepines makes it difficult to distinguish between therapeutic use and misuse of these substances with a drug screen. Tests do not distinguish between single use, long-standing use, harmful use and dependence. They detect oxazepam and nordiazepam, the primary metabolites of most benzodiazepine drugs. After ingestion, highly lipophilic agents such as diazepam are detected within minutes in serum and within $36 \mathrm{~h}$ in the urine (Laloup 2007). Agents that are extensively metabolised with long half-lives (e.g. diazepam, chlordiazepoxide) can be detected in the urine for more than a week after ingestion (Table 2).

Studies have reported false-negative results with immunoassay screening for benzodiazepines compared with gas chromatography-mass spectrometry (GC-MS). This is the main reason for underestimating the problem of benzodiazepine misuse. An additional drawback of immunoassay is its inability to identify specific benzodiazepines (Borrey 2003). Many patients start using a single benzodiazepine after it has been prescribed on a temporary basis. Unfortunately, this can contribute to long-term misuse and dependence. Benzodiazepines are widely accessible on the black market and over the internet. Benzodiazepine withdrawal should be considered in patients presenting with anxiety symptoms.

The GC-MS method offers several advantages over immunoassay. It has better sensitivity and thus detects benzodiazepines at lower levels, when the

\section{TABLE 2 Drug detection times in urine}

\begin{tabular}{|ll|}
\hline Drug & Detection time \\
\hline Amphetamines and analogues & 2 days \\
\hline Benzodiazepines & \\
Ultra short acting (e.g. midazolam) & 12 hours \\
Short acting (e.g. triazolam) & 24 hours \\
Intermediate acting (e.g. temazepam, chlordiazepoxide) & $2-5$ days \\
Long acting (e.g. diazepam, nitrazepam) & 7 days or more \\
\hline Buprenorphine and metabolites & 8 days \\
\hline Cocaine metabolite & $2-3$ days \\
\hline Methadone (maintenance dosing) & $7-9$ days \\
\hline Codeine, dihydrocodeine, morphine, propoxyphene & 2 days \\
\hline Cannabinoids & \\
Single use & $3-4$ days \\
Moderate use (three times a week) & $5-6$ days \\
Heavy use (daily) & 20 days \\
Chronic heavy use (more than three times daily) & Up to 45 days \\
\hline
\end{tabular}

Source: Department of Health 2007 
assay fails. It identifies the type of benzodiazepine being used and therefore allows clinicians to detect if more than one type of benzodiazepine is being used. Hence it can be useful in separating prescription drugs from those that are acquired off the streets. If there is high index of suspicion that any of the above is happening, then GC-MS can help identify such cases.

\section{Cannabinoids (marijuana)}

Delta-9-tetrahydrocannabinol (delta 9-THC) is the most psychoactive chemical in the cannabis plant. Tetrahydrocannabinol has high lipid solubility. The extensive storage of the drug in body fat and its slow excretion lead to positive urine tests up to 4 days after even a single use of marijuana. Long-term use can produce positive results in the urine up to 45 days after cessation (Department of Health 2007).

Urine drug screens are designed to detect the primary metabolites of cannabis, especially 11-nor-delta-9-tetrahydrocannabinol-9-carboxylic acid (delta-9-carboxy-THC). Oral fluid testing can also be used. This offers the advantage of detecting delta-9-carboxy-THC directly, which is sequestered in the oral mucosa, rather than metabolites, which appear at a later stage. Thus, the oral fluid test can be used to indicate recent use when it is likely that the person is still experiencing the pharmacological effects of the drug. As seen with other drugs, GC-MS is more sensitive and has a longer detection window than screening tests (Niedbala 2001).

A commonly encountered problem is differentiating between active and passive smokers of cannabis, as even passive smokers can test positive for THC. The difference is that active smokers have a biphasic pattern of decline in oral fluid THC concentration, whereas passive smokers have a linear decline. It is argued that the initial peak is due to the local sequestration of THC and the second peak is due to secretion into the oral fluid from the THC absorbed into the blood stream, which happens to a greater extent in active smokers. For the same reason, urine drug screens are more likely to be positive in active smokers (Niedbala 2004). Also, the saliva test remains positive for only around $30 \mathrm{~min}$ after passive ingestion, unlike in active smokers where it remains positive for hours or even days.

\section{Cocaine}

Cocaine stimulates the central nervous system and is misused primarily for its euphoric effect. It is used through different routes: inhalation (snorting), oral, intravenous and smoked. It is metabolised by butyrylcholinesterase and liver esterase into inactive metabolites. It has up to eight metabolites, the main metabolite being benzoylecgonine. The half-life of a single dose of cocaine in blood is about 30-90 min. After brief periods of cocaine use the metabolite is detectable in urine for up to 3 days. Repeated dosing extends oral fluid detection times for cocaine approximately fourfold and benzoylecgonine detection times sevenfold, whereas urine benzoylecgonine detection is extended twofold (Jufer 2006). Immunoassay can detect only the main metabolite and it cannot differentiate between the various other metabolites, which can only be done by GC-MS. The latter also detects the metabolites at lower concentrations. The concurrent use of cocaine and alcohol may result in the accumulation of a distinct metabolite, cocaethylene, which is longer lasting and accounts for enhanced subjective effects and toxicity (Politi 2007). It is only detected by chromatography methods.

\section{Opioids}

The term opiate refers to naturally occurring or semi-synthetic drugs such as morphine, codeine and diamorphine (heroin), whereas the term opioid includes natural and completely synthetic agents such as methadone and buprenorphine which have morphine-like actions (e.g. analgesia, sedation, constipation, respiratory depression). Morphine and codeine are naturally occurring alkaloids from the opium poppy seed (Papaver somniferum).

\section{Opiate drug screens}

Most drug screens for opiates detect morphine, which is also the primary metabolite of heroin and codeine. Morphine is further metabolised to 3-morphine-glucuronide and 6-morphineglucuronide. The 3-morphine-glucuronide accounts for $50 \%$ of morphine in urine. About $10-15 \%$ of codeine is converted to nor-codeine and is also detected in urine.

One common problem with opiate drug screens (immunoassays) is that they tend not to detect completely synthetic agents such as methadone and buprenorphine, which are widely used in the treatment of opioid addiction. Moreover, they often cross-react with over-the-counter medications containing codeine and dihydrocodeine (e.g. cold cures, mild analgesics). Often, GC-MS is required to distinguish between these compounds.

Fentanyl and oxycodone are not detected in urine screens: the former because of lack of metabolites, and the latter because of its derivation from thebaine, a compound that is not detected in the urine (Purdue Pharma LP 2009). 
Heroin (diacetylmorphine) is more potent and has a more rapid onset of action than morphine. Heroin is rapidly metabolised to 6-monoacetylmorphine (6-MAM), morphine and morphine glucuronide. Heroin can be detected in the serum 3-5 min after ingestion, and the metabolite, morphine, up to 2-4 days after heroin use. Detection of 6-MAM by GC-MS is used as a confirmatory test for heroin use as it is not produced by morphine or codeine. Unfortunately, as the 6-MAM metabolite has a short half-life of $36 \mathrm{~min}$, it is detected in the urine only up to $8 \mathrm{~h}$ after heroin use (Cone 1993).

\section{Opioid drug screens}

Methadone Methadone is a long-acting synthetic opioid that is used as substitution treatment for opioid dependence and chronic pain. About a third of the drug is excreted unchanged. Assays are designed to detect the parent compound and hence are specific. There is no need for a confirmatory test. The standard screening procedure is designed to detect the presence of the primary methadone metabolite EDDP in urine. In individuals where EDDP is detected by the specific initial immunoassay screen and where they are known to have been prescribed methadone, no further confirmatory testing needs to be performed. In individuals where methadone is not prescribed but EDDP is detected, or in those thought to be nonadherent and adding methadone to their urine, specimens need to be screened for the presence of both methadone and EDDP. It is therefore important to indicate whether or not methadone is prescribed, or if adulteration of urine specimen by the individual is suspected (Lancelin 2005).

Buprenorphine Buprenorphine is a long-acting synthetic opioid that is also used as substitution treatment for opioid dependence. It can also be combined with the opiate antagonist naloxone to treat opiate addiction. Buprenorphine undergoes extensive first-pass metabolism and therefore has very low oral bio-availability; however, its bioavailability sublingually is extensive enough to make this a feasible route of administration for the treatment of opioid dependence.

The mean time to maximum plasma concentration following sublingual administration is variable, ranging from $40 \mathrm{~min}$ to $3.5 \mathrm{~h}$. Buprenorphine has a large volume of distribution and is highly protein bound (96\%). It is extensively metabolised to norbuprenorphine (the main metabolite) primarily through cytochrome P450 (CYP) 3A4. The terminal elimination half-life of buprenorphine is long and there is considerable variation in reported values (mean values ranging from 3 to $44 \mathrm{~h}$ ). Most of a dose of buprenorphine is eliminated in the faeces, with about $10-30 \%$ excreted in urine. The presence of naloxone does not appear to influence the pharmacokinetics of buprenorphine (Elkader 2005). Even though the GC-MS method is the most accurate and specific, more recent assay methods are fairly specific (only $1 \%$ cross-reactivity with other opiods) and sensitive (95\% accuracy) (Wang 2007). Hence an assay is adequate in the mental health setting.

\section{Analysis}

\section{Cut-off levels}

The presence of a particular drug itself does not in itself constitute a positive result for substance misuse. Tests can be oversensitive. They can also detect very low levels of substances that cross-react with the tests for illicit drugs (e.g. over-the-counter painkillers containing codeine, bread containing poppy seeds). This has led to the adoption of cut-off levels above which it is very unlikely that other naturally occurring compounds will crossreact and the specimen can be reliably declared to indicate use of illegal drugs. This differs from the 'sensitivity' or 'detection limit' of the test, which is the absolute lowest detectable analyte concentration that will produce a positive result in a multitude of circumstances besides illicit drug use (e.g. occupational exposure to illicit drugs or 'passive smoking' of cannabis) (Saxon 1988).

In North America, cut-off levels in the Federal workplace drug-testing programmewere established in the mid-1980s. In the UK, even though there are no mandatory requirements for cut-off levels, most laboratories follow those recommended by the US Substance Abuse and Mental Health Services Administration (SAMHSA) (Parliamentary Office of Science and Technology 2004).

\section{Interpretation}

\section{When to order a drug test}

Illicit drug use can cause physical, mental and behavioural disturbances which may mimic psychiatric disorders. Therefore it needs to be considered in the differential diagnosis. A drug test aids in moving from a provisional to a confirmed diagnosis. If the history, mental status examination and physical examination are consistent with each other and suggest illicit drug use, a screening test (immunoassay) is sufficient to confirm the diagnosis after other cross-reacting products (see below) are ruled out. If there is a diagnostic dilemma, one can order a confirmatory test (chromatography). A positive screen does not constitute illicit drug use. There are numerous products that can crossreact with the test and cause false positives, 
including psychotropic medications. This can lead to unnecessary disagreements with the patient and destroy therapeutic alliance. When in doubt, a confirmatory test is invaluable. A confirmatory test may also be ordered when the patient requests one because they dispute the results of the screening test.

\section{False-positive results}

If a person takes an illicit drug and the test result is positive, then the test result is declared as a 'true positive'. If, however, the patient has not taken the drug but has a positive test result then the test result is declared as 'false positive' (Box 3).

\section{Amphetamine}

The common Vicks ${ }^{\oplus}$ nasal inhaler contains an isomer of amphetamine and cross-reacts with older immunoassay tests when used in large quantities. Newer EMIT tests however do not show positive results with the Vicks ${ }^{\circledast}$ nasal inhaler even when used up to twice the recommended dose (Poklis 1995). Many over-the-counter medications contain pseudoephedrine, phenylpropanolamine, ephedrine and phenylephrine. Cross-reactivities for these medications are greater than reported by the manufacturer (Stout 2004). AntiParkinsonian drugs like selegiline and deprenyl produce l-amphetamine and l-methamphetamine metabolites, which give a positive result on immunoassays (Romberg 1995). Unfortunately, a routine chromatography will not distinguish between the two isomers and it requires chiral chromatography to differentiate between the d- and l-forms (Cody 2002).

B0X 3 Agents with potential to cause false positives in immunoassay

\begin{tabular}{|c|c|}
\hline Amphetamine & Cocaine \\
\hline \multirow{5}{*}{$\begin{array}{l}\text { Amantadine, amphetamine analogues, } \\
\text { benzathine penicillin, bupropion, } \\
\text { chlorpromazine, deprenyl, desipramine, } \\
\text { ephedrine, labetalol, mebeverine, } \\
\text { methylphenidate, perazine, phenothiazines, } \\
\text { phenylephrine, phenylpropanolamine, } \\
\text { promethazine pseudoephedrine, ranitidine, } \\
\text { ritodrine, selegiline thioridazine, trazodone, } \\
\text { trimipramine }\end{array}$} & $\begin{array}{l}\text { Derivatives of coca plant, topical } \\
\text { anaesthetics }\end{array}$ \\
\hline & Opioids \\
\hline & $\begin{array}{l}\text { Codeine, poppy seeds, quinolones, } \\
\text { rifampicin, verapamil }\end{array}$ \\
\hline & Phencyclidine \\
\hline & $\begin{array}{l}\text { Diphenhydramine, ibuprofen, ketamine, } \\
\text { thioridazine, tramadol, venlafaxine }\end{array}$ \\
\hline Benzodiazepines & Methadone \\
\hline Oxaprozin, sertraline & Phenothiazines, clomipramine, verapamil, \\
\hline Cannabis & \\
\hline $\begin{array}{l}\text { Non-steroidal anti-inflammatory drugs, } \\
\text { efavirenz, proton pump inhibitors, hemp- } \\
\text { containing food items }\end{array}$ & \\
\hline
\end{tabular}

\section{Amphetamine} chlorpromazine, deprenyl, desipramine ephedrine, labetalol, mebeverine, phenylephrine, phenylpropanolamine, promethazine pseudoephedrine, ranitidine, itodrine, selegiline thioridazine, trazodone,

\section{Benzodiazepines}

Cannabis

containing food items
Medication used in attention-deficit hyperactivity disorder and narcolepsy and certain anorexiants produce false-positive results for drug misuse because they contain amphetamine (Manzi 2002).

Psychotropic medications such as bupropion (Weintraub 2000), phenothiazines (e.g. chlorpromazine, promethazine, thioridazine) (Olsen 1992), trazodone (Roberge 2001) and tricyclic antidepressants (Merigian 1993) have all been reported to interfere with immunoassays. Other agents found to cross-react with the amphetamine immunoassay include the antihypertensive labetalol (Gilbert 1995), the anti-ulcer drug ranitidine (Poklis 1991), the tocolytic drug ritodrine (Nice 1989), the antibiotic drug benzathine penicillin (Garcia 1998) and the antispasmodic drug mebeverine (Elliot 2006).

For this reason it is important to note the patient's medication and consider the possibility of false positives if they are using any of the above agents. If they are a known drug user and on one of the medications above, a confirmatory test is indicated to ascertain the source of the positive result in a screening test.

\section{Benzodiazepine}

Oxaprozin is a non-steroidal anti-inflammatory drug (NSAID) marketed for treatment of rheumatoid arthritis and osteoarthritis. It crossreacts with benzodiazepine (Fraser 1998). Older assay techniques cross-reacted with sertraline and its metabolites, but the newer, improved CEDIA benzodiazepine assay eliminates cross-reactivity (Fitzgerald 1997).

Aproblemthatisencounteredinbothmentalhealth and substance misuse clinics is the misuse of both prescribed and non-prescribed benzodiazepines. In individuals prescribed benzodiazepines, an assay does not give any additional information. It does not detect the different types of benzodiazepines and therefore is unable to inform us whether more than one benzodiazepine is being used. Multiple benzodiazepine misuse is common and chromatography methods are the only way of detecting the use of benzodiazepines that are not prescribed (Garretty 1997).

Cannabis

Non-steroidal anti-inflammatory drugs have been reported to interfere and cause false-positive results for cannabis assays, although conflicting results have been reported (Rollins 1990; Joseph 1995). It is speculated that NSAIDs interfere with the enzyme on the EMIT tests, leading to falsepositive results. Other agents that have been shown 
to cross-react with cannabinoid immunoassays include efavirenz (la Porte 2006), a non-nucleoside reverse transcriptase inhibitor used to treat HIV/ AIDS, and some proton pump inhibitors used for peptic ulcer disease (Wyeth 2009).

In the past, there have been concerns raised about testing positive for cannabis through passive exposure and consuming certain food items. Studies have shown that it is highly unlikely for an individual to test positive for THC by urine immunoassay through passive exposure (PerezReyes 1983). More recent urine testing procedures ensure that cannabinoids from foods (e.g. hempseed tea, hemp-seed oil) are beneath the cut-off concentrations for both EMIT and GC-MS tests (Steinagle 1999).

\section{Cocaine}

Urine screens for cocaine are very accurate in detecting recent cocaine ingestion. Previously, amoxicillin has been causally linked to falsepositive urine drug screens for cocaine metabolites. More recent reports conclude that this is unlikely (Reisfield 2008). Coca tea, derived from the same plant as cocaine, is commonly ingested in South America. This and other natural products derived from coca plant leaves produce positive cocaine screen results (Mazor 2006). Positive results in urine samples can also be seen in children exposed to cocaine in heavily contaminated environments (De Giorgio 2004).

\section{Opioids}

Ingestion of over-the-counter medications that contain codeine (e.g. cough medicines, medications for diarrhoea) must be excluded before confirming opioid misuse. As suggested, poppy seed ingestion can also lead to positive results and hence the US Department of Health and Human Services raised the cut-off level in 1998. Rifampicin, a drug usually used in the treatment of tuberculosis, interferes with opiate immunoassays (Daher 2002). Other drugs that contribute to false-positive urine screens for opiates are the quinolone group of antibiotics (Baden 2001) and cardiac drugs such as verapamil (Lichtenwalner 1998).

BOX 4 Clinical features of illicit drug use

- Illicit drug use can present in A\&E departments, acute psychiatric wards, and drug and alcohol clinics

- Illicit drugs can mimic and exacerbate psychiatric disorders

- Illicit drugs can confound diagnosis and treatment
TABLE 3 Comparison of test types

\begin{tabular}{|c|c|c|c|}
\hline Test type & Primary method & Strengths & Weaknesses \\
\hline Screening test & Immunoassay & $\begin{array}{l}\text { Near-patient or on-the- } \\
\text { spot tests } \\
\text { Require little expertise } \\
\text { Sensitive } \\
\text { Relatively inexpensive }\end{array}$ & Many false positives \\
\hline Confirmatory test & Chromatography & $\begin{array}{l}\text { Very sensitive } \\
\text { Very specific }\end{array}$ & $\begin{array}{l}\text { Expensive } \\
\text { Requires expert } \\
\text { laboratory analysis }\end{array}$ \\
\hline
\end{tabular}

\section{Methadone}

Psychotropic drugs such as phenothiazines (levomepromazine, chlorpromazine), clomipramine and thioridazine can cause a positive test result. False-positive results for methadone have been reported with other techniques and were attributable to metabolites of verapamil, diphenhydramine and doxylamine (Lancelin 2005).

\section{Conclusions}

Illicit drug use and its detection have important ramifications in general mental health services (Box 4). Psychiatrists need to be more proactive in requesting tests in a scientific manner based on drug detection windows, which will in turn depend on the specimen used and the drug in question. Although urine continues to be the most commonly used specimen, oral fluids have many advantages and should be considered more often. Ideally, analysis of specimens should look at metabolites rather than the parent drug, as presence of the latter could indicate external contamination rather than ingestion.

On-the-spot or near-patient tests are screening tests: they are convenient and quick but have the potential for both under- (false negatives) and overreporting (false positives) the presence of drugs (Table 3). For many reasons screening tests will continue to be used widely until there are easier-to-use, less expensive confirmatory tests. It is therefore very important that psychiatrists gain competence in interpreting these tests. A wide array of benign substances, including common over-the-counter medications, can produce falsepositive results. This should inform diagnostic formulations, as erroneous labelling can be even more serious than missing the diagnosis.

\section{References}

Armbruster DA, Schwarzhoff RH, Hubster EC, et al (1993) Enzyme immunoassay, kinetic microparticle immunoassay, radioimmunoassay, and fluorescence polarization immunoassay compared for drugs-of-abuse screening. Clinical Chemistry 39: 2137-46.

Baden LR, Horowitz G, Jacoby H, et al (2001) Quinolones and falsepositive urine screening for opiates by immunoassay technology. JAMA 286: $3115-9$ 


\section{MCO answers \\ 1 b $\quad 2$ a $\quad 3 a \quad 4 b \quad 5 e$}

Borrey D, Meyer E, Duchateau L, et al (2003) Longitudinal study on the prevalence of benzodiazepine misuse in a prison: importance of analytical strategy. Addiction 98: 1427-32.

Casavant MJ (2002) Urine drug screening in adolescents. Pediatric Clinics of North America 49: 317-27.

Cody JT (2002) Precursor medications as a source of methamphetamine and/or amphetamine positive drug testing results. Journal of Occupational and Environmental Medicine 44: 435-8.

Cone EJ, Dickerson S, Paul BD, et al (1993) Forensic drug testing for opiates. V. Urine testing for heroin, morphine, and codeine with commercial opiate immunoassays. Journal of Analytical Toxicology 17: 156-64.

Crome IB, Bloor R, Thom B (2006) Screening for illicit drug use in psychiatric hospitals: whose job is it? Advances in Psychiatric Treatment 12: $375-83$.

Daher R, Haidar JH, Al-Amin H (2002) Rifampin interference with opiate immunoassays. Clinical Chemistry 48: 203-4.

De Giorgio F, Rossi SS, Rainio J, et al (2004) Cocaine found in a child's hair due to environmental exposure? International Journal of Legal Medicine 118: 310-2.

Department of Health (2007) Drug Misuse and Dependence: UK Guidelines on Clinical Management. Department of Health (http:// www.nta.nhs.uk/uploads/clinical_guidelines_2007.pdf).

Dolan K, Rouen D, Kimber J (2004) An overview of the use of urine, hair, sweat and saliva to detect drug use. Drug Alcohol Review 23: 213-7.

Drummond C, Ghodse H (1999) Use of investigations in the diagnosis and management of alcohol use disorders. Advances in Psychiatric Treatment 5: 366-75

Elkader A, Sproule B (2005) Buprenorphine: clinical pharmacokinetics in the treatment of opioid dependence. Clinical Pharmacokinetics 44 $661-80$

Elliott S, Burgess V (2006) Investigative implications of the instability and metabolism of mebeverine. Journal of Analytical Toxicology 30 : $91-7$

Eskridge KD, Guthrie SK (1997) Clinical issues associated with urine testing of substances of abuse. Pharmacotherapy 11: 491-510.

Fitzgerald RL, Herold DA (1997) Improved CEDIA benzodiazepine assay eliminates sertraline cross reactivity. Journal of Analytical Toxicology 21: $32-5$.

Fraser AD, Howell P (1998) Oxaprozin cross-reactivity in three commercial immunoassays for benzodiazepines in urine. Journal of Analytical Toxicology 22: 50-4.

Garcia Callejo FJ, Velert Vila MM, Costa I, et al (1998) Unusual analytical interference caused by benzathine penicillin G. Acta Otorrinolaringológica Española 49: 671-2.

Garretty DJ, Wolff K, Hay AW, et al (1997) Benzodiazepine misuse by drug addicts. Annals of Clinical Biochemistry 34 (pt 1): 68-73.

Gilbert RB, Peng PI, Wong D (1995) A labetalol metabolite with analytical characteristics resembling amphetamines. Journal of Analytical Toxicology 19: 84-6.

Home Office (2006) Drug Misuse Declared: Findings from the 2005/06 British Crime Survey. England and Wales. Home Office.

Hsu J, Liu C, Liu CP, et al (2003) Performance characteristics of selected immunoassays for preliminary test of 3,4-memylenedioxymetluimphetamine, methamphetamine, and related drugs in urine specimens. Journal of Analytical Toxicology 27: 471-8.

Jaffee WB, Trucco E, Levy S, et al (2007) Is this urine really negative? A systematic review of tampering methods in urine drug screening and testing. Journal of Substance Abuse Treatment 33: 33-42.

Joseph R, Dickenson S, Willis R, et al (1995) Interference by nonsteroidal anti-inflammatory drugs in EMIT and TDx assays for drugs of abuse. Journal of Analytical Toxicology 19: 13-7.
Jufer R, Walsh S L, Cone E J, et al (2006) Effect of repeated cocaine administration on detection times in oral fluid and urine. Journal of Analytical Toxicology 30: 458-62.

Kim I, Barnes AJ, Oyler JM, et al (2002) Plasma and oral fluid pharmacokinetics and pharmacodynamics after oral codeine administration. Clinical Chemistry 48: 1486-96.

la Porte CJ, Droste JA, Burger DM (2006) False-positive results in urine drug screening in healthy volunteers participating in phase 1 studies with efavirenz and rifampin [letter]. Therapeutic Drug Monitoring 28. 286

Laloup M, Ramirez Fernandez MD, Wood M, et al (2007) Detection of diazepam in urine, hair and preserved oral fluid samples with LC-MS-MS after single and repeated administration of Myolastan and Valium. Analytical and Bioanalytical Chemistry 388: 1545-56.

Lancelin F, Kraoul L, Flatischler N, et al (2005) False-positive results in the detection of methadone in urines of patients treated with psychotropic substances. Clinical Chemistry 51: 2176-7.

Lichtenwalner MR, Mencken T, Tully R, et al (1998) False-positive immunochemical screen for methadone attributable to metabolites of verapamil. Clinical Chemistry 44: 1039-41.

Manzi S, Law T, Shannon MW (2002) Methylphenidate produces a false-positive urine amphetamine screen. Pediatric Emergency Care 18: 401.

Mazor SS, Mycyk MB, Wills BK, et al (2006) Coca tea consumption causes positive urine cocaine assay. European Journal of Emergency Medicine 13: 340-1.

Merigian KS, Browning R, Kellerman A (1993) Doxepin causing falsepositive urine test for amphetamine. Annals of Emergency Medicine 22: 1370 .

Nice A, Maturen A (1989) False-positive urine amphetamine screen with ritodrine. Clinical Chemistry 35: 1542-3.

Niedbala RS, Kardos KW, Fritch DF, et al (2001) Detection of marijuana use by oral fluid and urine analysis following single-dose administration of smoked and oral marijuana. Journal of Analytical Toxicology 25: 289-303.

Niedbala S, Kardos K, Salamone S, et al (2004) Passive cannabis smoke exposure and oral fluid testing. Journal of Analytical Toxicology 28. $546-52$

OIsen KM, Gulliksen M, Christophersen AS (1992) Metabolites of chlorpromazine and brompheniramine may cause false-positive urine amphetamine results with monoclonal EMIT d.a.u. immunoassay. Clinical Chemistry 38: 611-2.

Parliamentary Office of Science and Technology (2004) Postnote. Drug Tests. September 2004, Number 228. Parliamentary Office of Science and Technology (http://www.parliament.uk/documents/upload/ POSTpn228.pdf).

Peace MR, Tarnai LD (2002) Performance evaluation of three on-site adulterant detection devices for urine specimens. Journal of Analytical Toxicology 26: 464-70.

Pehrsson A, Gunnar T, Engblom C, et al (2008) Roadside oral fluid testing: comparison of the results of drugwipe 5 and drugwipe benzodiazepines on-site tests with laboratory confirmation results of oral fluid and whole blood. Forensic Science International 175: $140-8$.

Perez-Reyes M, Di Guiseppi S, Mason AP, et al (1983) Passive inhalation of marihuana smoke and urinary excretion of cannabinoids. Clinical Pharmacology and Therapeutics 34: 36-41.

Poklis A, Hall KV, Still J, et al (1991) Ranitidine interference with the monoclonal EMIT d.a.u. amphetamine/methamphetamine immunoassay. Journal of Analytical Toxicology 15: 101-3.

Poklis A, Moore KA (1995) Response of EMIT amphetamine immunoassays to urinary desoxyephedrine following Vicks inhaler use. Therapeutic Drug Monitoring 17: 89-94. 
Politi L, Zucchella A, Morini L, et al (2007) Markers of chronic alcohol use in hair. Comparison of ethyl glucuronide and cocaethylene in cocaine users. Forensic Science International 172: 23-7.

Purdue Pharma LP (2009) OxyContin Package Insert. Purdue Pharma LP (http://www.purduepharma.com/pi/prescription/oxycontin.pdf).

Reisfield GM, Haddad J, Wilson GR, et al (2008) Failure of amoxicillin to produce false-positive urine screens for cocaine metabolite. Journal of Analytical Toxicology 32: 315-8.

Roberge RJ, Luellen JR, Reed S (2001) False-positive amphetamine screen following a trazodone overdose. Journal of Toxicology: Clinical Toxicology 39: 181-2

Rollins DE, Jennison TA, Jones G (1990) Investigation of interference by nonsteroidal anti-inflammatory drugs in urine tests for abused drugs. Clinical Chemistry 36: 602-6.

Romberg RW, Needleman SB, Snyder JJ, et al (1995) Methamphetamine and amphetamine derived from the metabolism of selegiline. Journal of Forensic Sciences 40: 1100-2.

Saxon AJ, Calsyn DA, Haver VM, et al (1988) Clinical evaluation and use of urine screening for drug abuse. Western Journal of Medicine 149: 296-303

Steinagle GC, Upfal M (1999) Concentration of marijuana metabolites in the urine after ingestion of hemp seed tea. Journal of Occupational and Environmental Medicine 41: 510-3.
Stout PR, Klette KL, Horn CK (2004) Evaluation of ephedrine, pseudoephedrine and phenylpropanolamine concentrations in human urine samples and a comparison of the specificity of DRI amphetamines and Abuscreen online (KIMS) amphetamines screening immunoassays. Journal of Forensic Sciences 49: 160-4.

United States Department of Transportation (2009) Urine Specimen Collection Guidelines for the US Department of Transportation Workplace Drug Testing Programs (revised). Office of Drug and Alcohol Policy and Compliance (http://www.dot.gov/ost/dapc/udsc.html).

Wang G, Vincent M, Rodrigues W, et al (2007) Development and GC$\mathrm{MS}$ validation of a highly sensitive recombinant G6PDH-based homogeneous immunoassay for the detection of buprenorphine and norbuprenorphine in urine. Journal of Analytical Toxicology 31: 377-82.

Warner A (1989) Interference of common household chemicals inimmunoassay methods for drugs of abuse. Clinical Chemistry 35 : 648-51.

Weintraub D, Linder MW (2000) Amphetamine positive toxicology screen secondary to bupropion. Depression and Anxiety 12: 53-4.

Workplace Drug Testing Forum (2001) United Kingdom Laboratory Guidelines for Legally Defensible Workplace Drug Testing: Urine Drug Testing. Version 1.0. WDT Forum (http://www.Itg.uk.net/admin/files/ WPDT_guidelines.pdf).

Wyeth (2009) Protonix: Full Prescribing Information. Wyeth (http:// www.wyeth.com/content/showlabeling. asp?id=135).

\section{MCQs}

Select the single best option for each question stem

\section{Regarding methods of drug testing:}

a urine testing is the most easy to administer, reliable and donor-friendly test

b oral fluid is convenient to collect, hard to adulterate and donor friendly

c hair testing has a short detection window

d sweat is used to test a wide variety of drugs

e the most commonly used sample in mental health settings is sweat.

2 The following are types of immunoassays:

a enzyme-multiplied immunoassay technique (EMIT)

b high performance liquid chromatography

c magnetic resonance imaging d gas chromatography-mass spectrometry

$$
\text { (GC-MS) }
$$

e near-patient tests.

3 In a healthy sample of urine

a the temperature is between $32^{\circ} \mathrm{C}$ and $38^{\circ} \mathrm{C}$

$\mathrm{b}$ the $\mathrm{pH}$ is between 2 and 4.5

c the specific gravity is usually less than 1

$d$ the creatinine concentration is less than $5 \mathrm{mg} / \mathrm{dl}$

e urinary nitrite levels should be less than $500 \mu \mathrm{g} / \mathrm{ml}$
4 A medication that can potentially cause false-positive results with amphetamine assays is:
a olanzapine
b chlorpromazine

\section{c poppy seeds \\ d ketamine \\ e venlafaxine.}

5 In interpreting the test results:

a the presence of drugs in a sample is sufficient to conclude drug misuse

b cut-off level and sensitivity are the same

c a single use of cannabis shows a positive test result for 30 days after use

d drug testing across Europe is standardised and results are easily comparable

e a detailed history is vital for accurate interpretation of tests. 